\title{
Successful Emergency Treatment in Bradypus variegatus following Non-Hemorrhagic Hypovolemic Shock
}

\author{
Marina Sette Camara Benarrós', Louysse Helene Monteiro', Sandy Kelly Souza Marques da Silva', \\ Cinthia Távora de Albuquerque Lopes' ${ }^{1}$ Sheyla Farhayldes Souza Domingues ${ }^{1,2}$
}

\begin{abstract}
Background: The brown-throated sloth (Bradypus variegatus) occurs from the Nicaragua to Brazil. In brazilian amazonian, these animals are rescued with illnesses caused by anthropic actions. Emergencial treatment of sloths is complex because is a lack of specie-specific information allied to a particularly physiology. They have low metabolism and physiological parameters during resting time is 4-7 breaths $/ \mathrm{min}, 40-100$ heartbeats $/ \mathrm{min}$ and temperature between $32-35^{\circ} \mathrm{C}$. They are also folivores, predisposing cases of dehydration and even hypovolemic shock. This study aimed to report the emergency treatment performed on a specimen of Bradypus variegatus affected by hypovolemic shock due to dehydration.

Case: A male sloth weighing $2.7 \mathrm{~kg}$ was attended at the Veterinary Hospital-Wild Animals Sector of the Federal University of Pará (HVSAS-UFPA), victim of untreated hypovolemic shock due three days of forced eating with inadequate food, which resulted in dehydration, non-hemorrhagic hypovolemic shock, and severe apathy. Physiological parameters were 8 breaths $/ \mathrm{min}$, heart rate $90 / \mathrm{min}$ and rectal temperature of $33^{\circ} \mathrm{C}$. Treatment begun with fluid therapy taking account of $10 \%$ of body dehydration ( $270 \mathrm{~mL}$ Ringer lactate, $8 \mathrm{~mL}$ vitamin complex and $2 \mathrm{~mL}$ glucose), for respiratory decompensation nebulization was conducted with epinephrine $(0.5 \mathrm{~mL} / \mathrm{kg})$, and intramuscular administration of vitamin B complex $(1 \mathrm{mg} /$ $\mathrm{kg})$, metoclopramide $(0.2 \mathrm{mg} / \mathrm{kg})$, ranitidine $(0.5 \mathrm{mg} / \mathrm{kg})$, and dexamethasone $(0.3 \mathrm{mg} / \mathrm{kg})$ to metabolic stimulation improvement. Physiological parameters were monitored every $10 \mathrm{~min}$ for the first $12 \mathrm{~h}$ of hospitalization. The management were gradually established as the clinical conditions were improved, including forced feeding, daily walks, and enclosure adapted for the minimal behavioral and biological requirements. After 14 days of intensive treatment, the animal was considered ready for release.

Discussion: Treatment of critically ill and unnourishment patients requires prompt interventions. Animals with low metabolism potentially have reduced chances of success, therefore, a prompt establishment of viable airways and body temperature maintenance would allow the efficient drugs metabolization. Severe dehydration can lead to animal death by hypovolemic shock, as a consequence of low blood volume, diminish in blood pressure and in the amount of oxygen in body supply, so fluid replacement is essential, as well as stimulating respiratory compensation through bronchodilator drugs. Furthermore, metabolic stimulation is important in cases to prevent numbness, which is common in very weak sloths. The constant monitoring of physiological parameters since at first moment of hospitalization provided parameter to decide about particular needs adaptations during the animal recovery time. Such protocols described for the brown-throated sloth was absent in the literature. In conclusion, the therapeutic and management protocol implemented and adapted to a particular patience, as brown-throated sloth, resulted in a gradual clinical improvement and allowed to its return for the natural environment.
\end{abstract}

Keywords: brown-throated sloth, wildlife, dehydration, hypovolemic shock.

Descritores: preguiça-comum, animal silvestre, desidratação, choque hipovolêmico. 


\section{INTRODUÇÃO}

A preguiça-comum (Bradypus variegatus, ordem Pilosa, superordem Xenarthra) é encontrada desde florestas da Nicarágua até a Mata Atlântica, no sul do Brasil [9]. Sua presença é comum em áreas antropizadas da Amazônia, e tem como principais ameaças atropelamentos, distribuições elétricas e caça para consumo humano $[1,26,27]$. Os espécimes resgatados, frequentemente enfermos, exigem atendimento clínico de emergência [17,22,24].

No caso das preguiças, o metabolismo lento dificulta a absorção de medicamentos intramusculares e subcutâneos, principalmente em casos de hipotermia/ hipoglicemia. Alternativas são os medicamentos tópicos, como agentes de nebulização, e intravenosos [10,19,22].

O gênero Bradypus, em repouso, mantém de 4 a 7 respirações/min, 40 a 100 batimentos cardíacos/min e temperatura entre 32 e $35^{\circ} \mathrm{C}[7,21]$. Tais parâmetros variam com a temperatura ambiental e hora do dia devido aos mecanismos de otimização da energia pela dieta pouco calórica [8]. Compensações ventilatórias, com aumentos fisiológicos da frequência respiratória (8 a 11 respirações/min), ocorrem durante alimentação, deslocamento e/ou estresse [7,21].

Por sua seletividade alimentar (folhas com alta densidade de água e consumo de água in natura), quadros de desidratação e inanição são comuns, principalmente em cativeiro, levando a perda de peso progressiva (em adultos considera-se o ideal de 3 a $5 \mathrm{~kg}$ ) e redução de eletrólitos predispondo choque hipovolêmico, com prejuízos a oxigenação e óbito [3,15,18-20].

O objetivo deste estudo foi relatar um protocolo terapêutico associado ao manejo em um espécime de preguiça-comum (Bradypus variegatus) acometido por choque hipovolêmico por desidratação.

\section{CASO}

Foi atendido no Hospital Veterinário - Setor de Animais Silvestres da Universidade Federal do Pará (HVSAS-UFPA) (Castanhal-PA) um indivíduo da espécie Bradypus variegatus proveniente de resgate pelo Batalhão de Bombeiros $\left(2^{\circ} \mathrm{GBM}\right)$, com histórico de criação domiciliar com alimentação forçada à base de banana e água misturados em liquidificador por três dias consecutivos.

No exame físico, foi identificado que era um macho adulto, pela presença de dimorfismo sexual (mancha amarela dorsal intraescapular, característica de animais com mais de 2 anos de idade) e realizada biometria, com balança digital, fita métrica e paquímetro de precisão, constatando-se peso de $2,7 \mathrm{~kg}$, circunferência da cabeça de $23 \mathrm{~cm}$, comprimento da cabeça de 7,5 cm, circunferência torácica de $32 \mathrm{~cm}$, circunferência abdominal de $37 \mathrm{~cm}$, garra direita com $5 \mathrm{~cm}$, garra esquerda com $4,8 \mathrm{~cm}$, comprimento total de $51 \mathrm{~cm}$ e comprimento da cauda $4 \mathrm{~cm}$.

$\mathrm{O}$ animal apresentava ausência de tônus muscular nos membros anteriores e posteriores, cabeça pendular, apoiada no esterno e sem capacidade de sustentação. Os parâmetros fisiológicos foram de 8 movimentos respiratórios/min, 90 batimentos cardía$\cos /$ min e temperatura retal de $33^{\circ} \mathrm{C}$.

Observou-se ainda desidratação, com tempo de retorno da prega cutânea acima de $3 \mathrm{~s}$, olhos opacos e fraqueza muscular. Alterações indicativas de choque hipovolêmico. Não havia presença de hemorragia externa nem de timpanismo (circunferência abdominal adequada ao padrão da espécie), as vias aéreas estavam desobstruídas e o animal estava taquipneico (8 a 9 respirações/ $\mathrm{min})$. Realizou-se tricotomia e acesso pela veia braquial (Figura 1) do membro anterior esquerdo para administração de fluidoterapia por gotejamento. O cálculo para fluidoterapia foi realizado considerando-se $10 \%$ de nível de desidratação, totalizando $270 \mathrm{~mL}$ (Ringer com lactato $^{\circledR 1}$ ) a serem realizados $50 \%$ no primeiro dia (135 $\mathrm{mL}$ ), e o restante dividido em dois dias subsequentes ( $25 \%$ ou $67,5 \mathrm{~mL} / \mathrm{dia}$ ). Foi adicionado à fluidoterapia $8 \mathrm{~mL}$ de complexo vitamínico $\left(\text { Polijet }^{\circledR}\right)^{2}$ e $2 \mathrm{~mL}$ de glicose $5 \%$ (Halexistar $\left.^{\circledR}\right)^{3}$ também no primeiro dia. Nos dias subsequentes foi administrado somente o volume de Ringer com lactato restante $(67,5 \mathrm{~mL} / \mathrm{dia}$, durante dois dias) e gradualmente o animal foi demonstrando o interesse no alimento, suspendendo-se a fluidoterapia.

Realizou-se nebulização por $10 \mathrm{~min}$ com 1 $\mathrm{mL}$ de Epinefrina ${ }^{\circledR} 4(0,5 \mathrm{~mL} / \mathrm{kg})$ diluída em $10 \mathrm{~mL}$ de $\mathrm{NaCl} 0,9 \%{ }^{\circledR 1}$ como auxiliar à compensação respiratória. Aplicação intramuscular de Complexo B (Hyplex B ${ }^{\circledR 5}$ $1 \mathrm{mg} / \mathrm{kg}$ ), Metoclopramida (Noprosil ${ }^{\circledR 6} 0,2 \mathrm{mg} / \mathrm{kg}$ ), Ranitidina (Cloridrato de raniditina ${ }^{\circledR 7} 0,5 \mathrm{mg} / \mathrm{kg}$ ) e Dexametasona (Dexaflan ${ }^{\circledR 8} 0,3 \mathrm{mg} / \mathrm{kg}$ ) como estimulantes metabólicos e indutores do apetite dado o histórico de alimentação inadequada e por este apresentar $300 \mathrm{~g}$ abaixo do peso mínimo indicado para a espécie.

Foram aferidos frequência cardíaca (FC) e frequência respiratória (FR), por auscultação torácica com estetoscópio (Littmann Classic III ${ }^{\circledR}$ ), e temperatura retal 


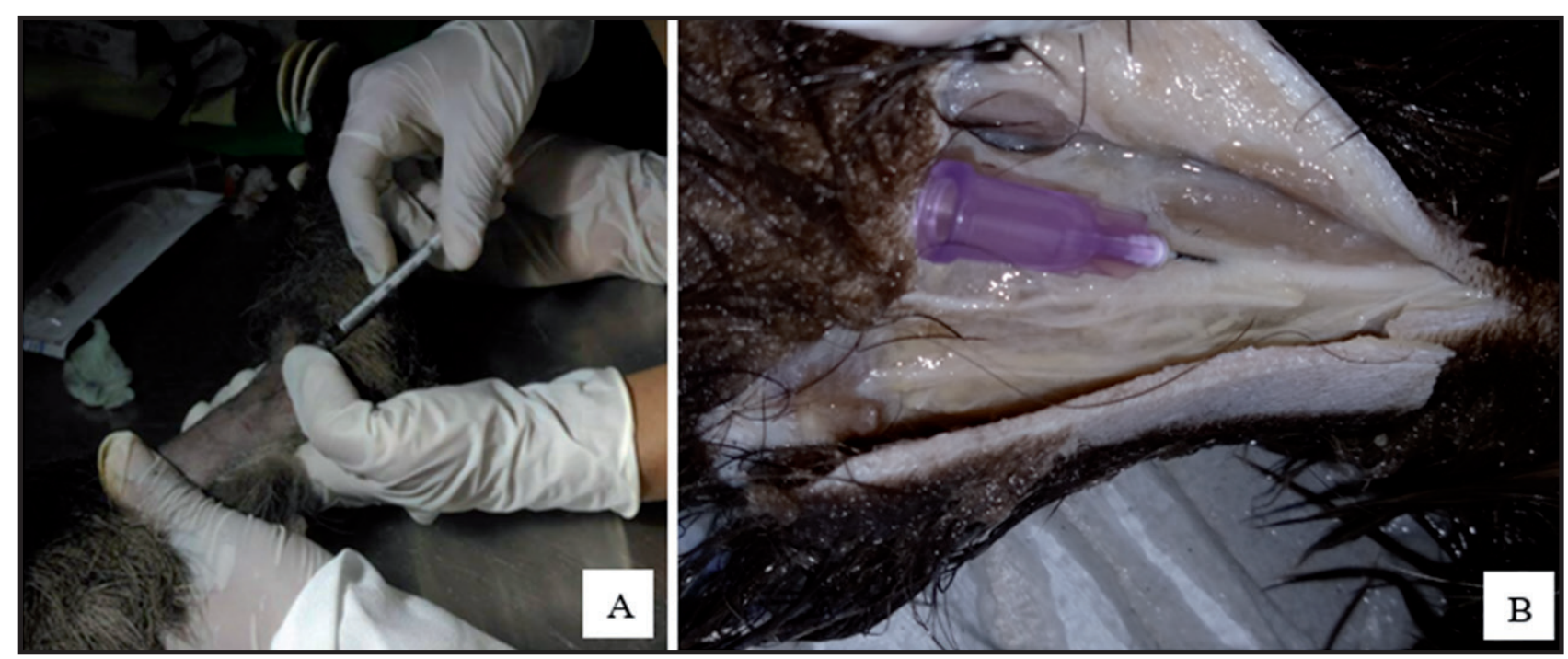

Figura 1. A- Acesso venoso pela veia braquial para administração de fluidoterapia no espécime. B- Posição anatômica da veia braquial do membro anterior, localizada na interseção dos músculos peitoral, deltóide e tríceps braquial em peça anatômica glicerinada

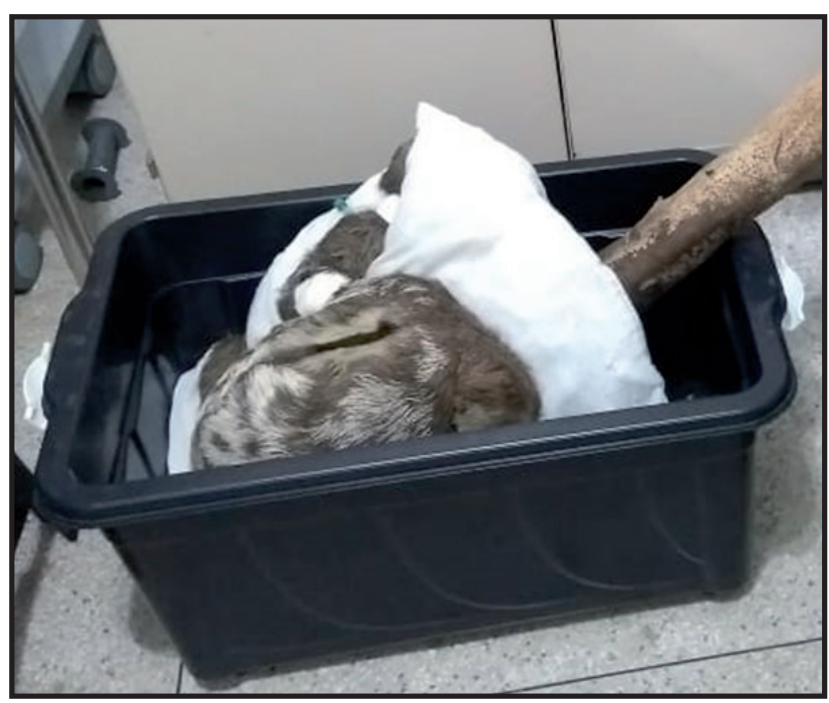

Figura 2. Animal mantido no ambulatório do HVSAS-UFPA durante a primeira noite de internação.

(TR) com termômetro digital (Gráfico 1), durante observação do animal a cada 10 min nas 12 primeiras h de internamento, consideradas as mais críticas (Figura 2).

Após $5 \mathrm{~h}$ da aplicação dos medicamentos (às 22 $\mathrm{h}$ da noite), observou-se ligeira redução da temperatura retal, possivelmente pela redução da temperatura do ambiente (de $29^{\circ} \mathrm{C}$ à $22-23^{\circ} \mathrm{C}$ ). Optou-se por aproximar o aquecedor do animal, o qual melhorou o quadro gradativamente, como observado no Gráfico 1 . As $12 \mathrm{~h}$ de observação (às $10 \mathrm{~h}$ da manhã do dia seguinte), a temperatura ambiental já era de cerca de $30^{\circ} \mathrm{C}$ e o animal foi considerado estável (TR 33,9 C FR 6 rpm e FC 90 bpm sem maiores variações e animal começou a movimentar-se).

Iniciaram-se, no dia seguinte, as medidas de manejo visando aumentar a atividade metabólica e movimentação (animal ainda sem se alimentar, sem defecar ou urinar desde sua entrada no HVSAS). Foram fornecidos na boca pedaços de embaúba (Cecropia sp.) umedecida, inicialmente forçado a cada $3 \mathrm{~h}$, até o animal demonstrar interesse pelo alimento. Ao segundo dia de internação, a alimentação passou a ser espontânea e animal foi mantido em recinto telado com galhos (próprio para reabilitação de preguiças, $1 \mathrm{x} 1 \mathrm{~m}$, feito de canos do tipo PVC, revestido por telas de tecido e plástico com galhos e cabos de vassoura em seu interior) [Figura 3]. O alimento era trocado 2 vezes ao dia e a água era fornecida ad libitum.

Foram estabelecidos banhos de sol e passeios em áreas gramadas, desde o primeiro dia se estendendo por todo o período de internação, de 15 a $20 \mathrm{~min}$, todos os dias pela manhã (8:00 h), a fim de se estimular a defecação, micção e liberação de gases (Figura 4). No terceiro dia de internamento o animal defecou e urinou (ambos de aspecto normal). Os momentos de maior atividade do animal (movimentos de escalagem, descida dos troncos, alimentação) eram no início da manhã e da tarde mesmo no recinto.

Paulatinamente, a partir do quarto dia de internação, o espécime foi retornando ao seu comportamento natural, alimentando-se sozinho, defecando e urinando nos passeios, a cada 5 dias em média, escalando em árvores durante o dia, e dormindo durante a noite estando apto à soltura 14 dias após sua chegada ao hospital e início do tratamento (Figura 5).

\section{DISCUSSÃO}

Animais silvestres podem apresentar certo subdesenvolvimento em regiões de antropização intensa, isto se deve principalmente a precariedade de acesso aos alimentos adequados e ausência de hábitats, além 


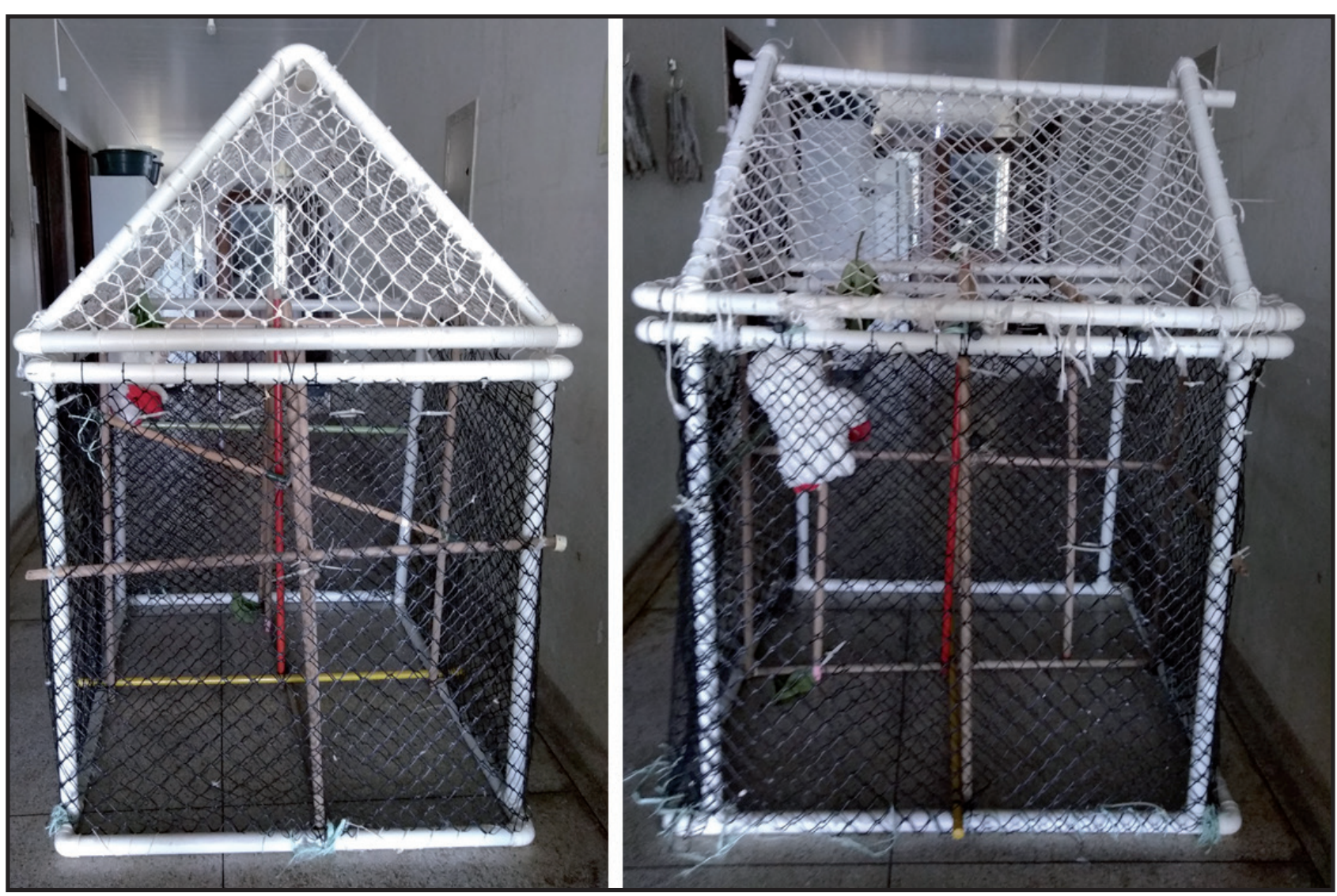

Figura 3. Recinto construído adaptado dentro do HVSAS para preguiças em reabilitação.

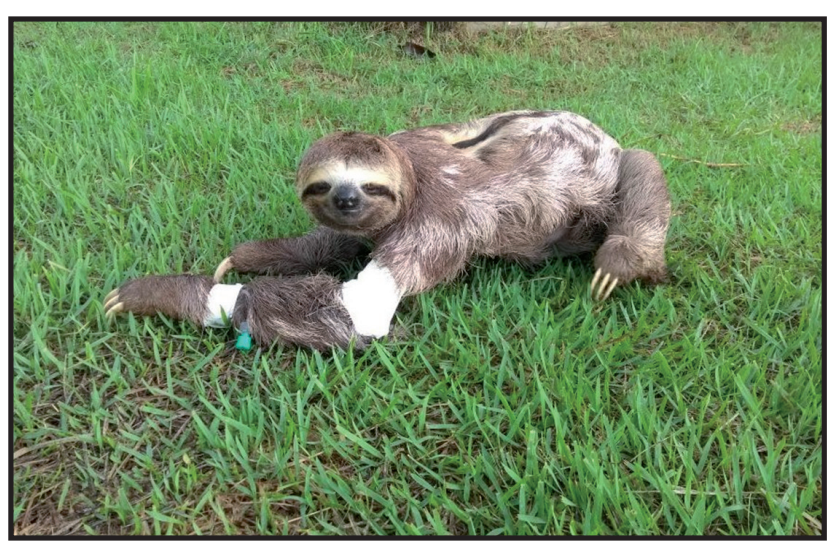

Figura 4. Animal em passeio pela manhã logo após ser considerado estável quanto aos parâmetros fisiológicos.

da maior predisposição a ocorrência de doenças. No espécime avaliado foi observado peso e comprimento menores que os indicados para a espécie em faixa etária correspondente, pois a presença de mancha dorsal intraescapular é o indicativo de que o animal já se encontra em maturidade sexual, ou seja, acima de 2 anos de idade. Indivíduos nessa fase de vida têm entre 60 e $70 \mathrm{~cm}$ de comprimento e mais de $3 \mathrm{~kg}[4,19]$.

$\mathrm{O}$ tratamento do paciente crítico e desnutrido exige intervenções rápidas e que garantam a sua sobrevida. No caso de animais de baixo metabolismo essa dificuldade é potencializada diminuindo as chances de sucesso do tratamento [7]. Desta forma, as primeiras medidas a serem tomadas são a viabilização das vias aéreas e a garantia de manutenção da temperatura corporal, principalmente em horas do dia com temperaturas mais amenas. Esta última permitirá a metabolização mais eficiente dos fármacos e outros compostos, além de prevenir o estado de torpor, esperado em preguiças mantidas em baixas temperaturas (termorregulação dependente do ambiente) $[8,13]$.

$\mathrm{O}$ animal em choque hipovolêmico pode apresentar sonolência, apatia e perda de tônus muscular dos membros devido à perda de eletrólitos suficientes para execução das funções orgânicas, frequentemente associados a medidas cardiorrespiratórias compensatórias (taquicardia, taquipneia). Consiste em um quadro de intervenção imediata com risco de óbito iminente se não corrigidos os fatores necessários (reposição de fluidos, oxigenação e manutenção da temperatura corporal) $[11,12,18]$.

A taquipneia é uma medida compensatória do sistema respiratório frente à hipóxia dos tecidos na presença de uma desordem circulatória [11]. A epinefrina é um agonista adrenérgico não seletivo de curta duração e rápida ação, amplamente empregada na pediatria humana como agente de nebulização em emergências respiratórias [14]. Isto se deve ao seu efeito broncodila- 


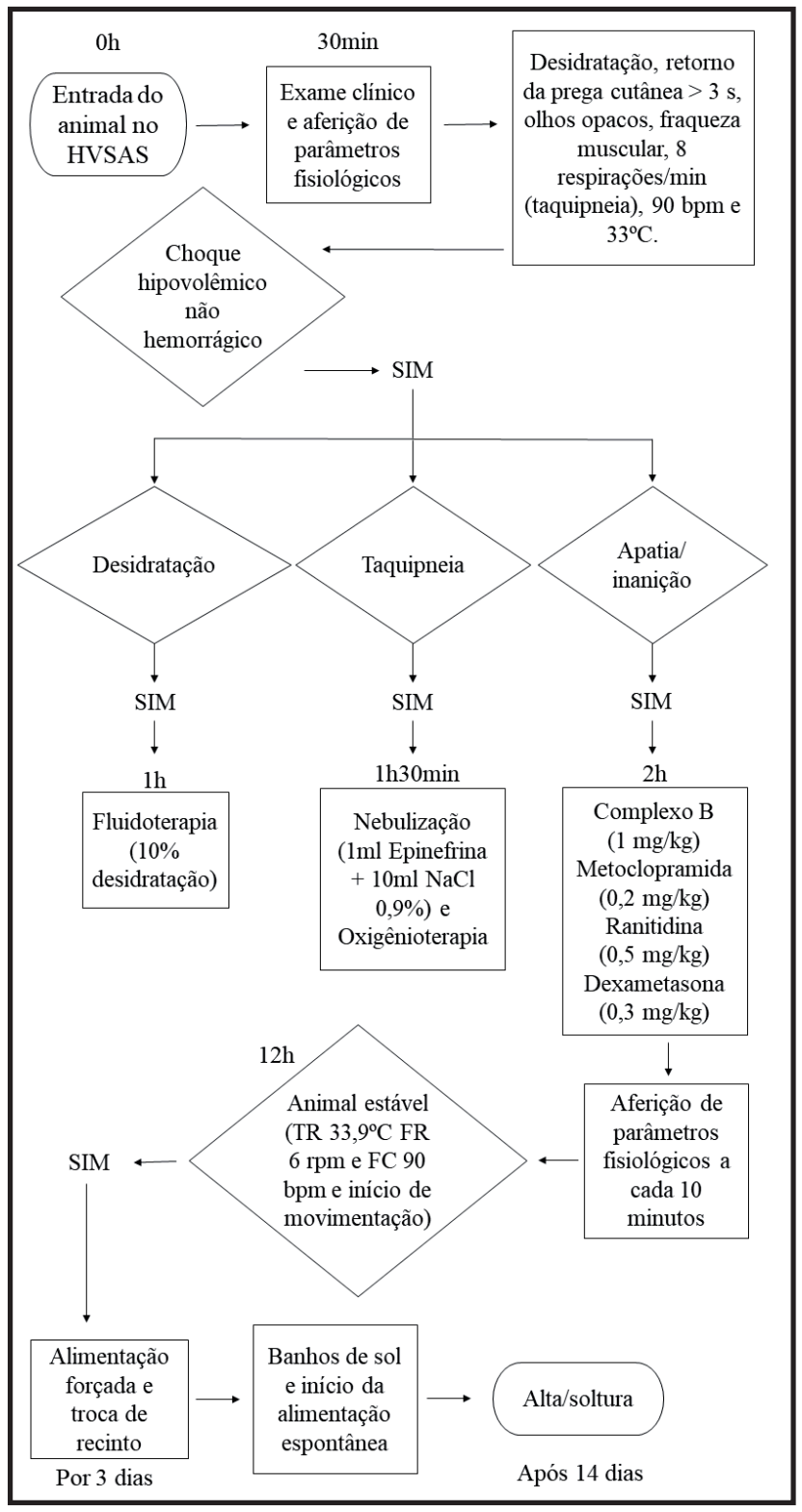

Figura 5. Fluxograma do atendimento emergencial e posterior manejo de Bradypus variegatus. $\square$ - início e resultados finais; $\square$ - processo; $>$ - tomadas de decisão.

tador imediato e vasodilatador quando usado de forma tópica nos pulmões [2,14]. Alternativa viável também para mamíferos em estados de hipoxemia generalizada.

Associados a epinefrina, os corticóides, como a dexametasona, também auxiliarão diminuindo os edemas inflamatórios de vias aéreas e estimulando o metabolismo farmacodinâmico, por meio de interações no metabolismo do ácido aracdônico, bloqueando a ação de enzimas como a Fosfolipase $\mathrm{A}_{2}$ e ciclo-oxigenases, diretamente relacionadas com a promoção da cascata inflamatória na membrana celular, e precursores de prostaglandinas, leucotrienos e tromboxanos. Existem relatos do uso deste fármaco em preguiças do gênero Bradypus principalmente em casos emergenciais $[13,14]$.

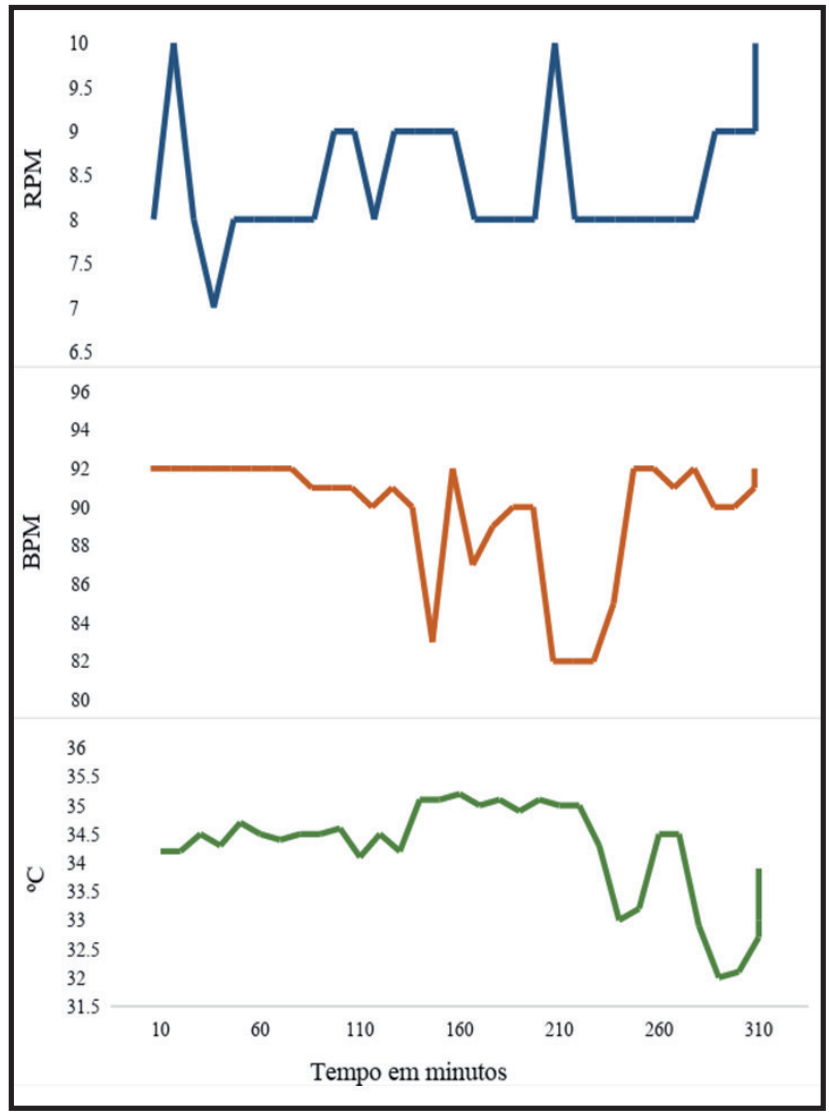

Gráfico 1. FR, FC e TR de B. variegatus em respirações por minuto aferidos a cada $10 \mathrm{~min}$.

Considerando-se ainda que preguiças são folívoras estritas com um trato gastrintestinal diferenciado, um animal em desidratação e anorexia é considerado de alta gravidade pela ausência de eletrólitos e glicose na corrente sanguínea, além de aumentar a predisposição ao timpanismo [6,16,25]. A fluidoterapia para reposição destes componentes também deve ser realizada no tempo e quantidade adequados ao animal, evitando-se desbalanceamento ácido básico [11]. Além disso, a estase intestinal é um dos principais precursores do timpanismo, então fármacos estimulantes da motilidade gástrica, como a metoclopramida e a ranitidina, também são incluídos na terapêutica de emergência nestes animais [3,23]. A presença de defecação e micção após o tratamento demonstra a viabilidade destes fármacos e o retorno da função renal que poderia estar prejudicada devido a desidratação.

Atrelados a aferição frequente dos parâmetros fisiológicos, essas são medidas que possibilitam uma estabilização inicial das funções básicas do organismo e permitem a intervenção rápida e precoce quando necessária (dispneias, reduções bruscas de temperatura, descompensações), tendo em vista a dificuldade de realização de procedimentos padronizados, como a 
intubação endotraqueal e esofagostomia, bem como a observação de alterações clínicas evidentes nesta espécie [5,25]. Outros fatores importantes à reabilitação são a adequação nutrição, manejo e reabilitação à biologia do animal, altamente específicos. Estes são pontos chave no sucesso do protocolo e garantia de reintrodução deste na natureza.

Concluiu-se, portanto, que o protocolo terapêutico foi eficiente e inovador na estabilização do quadro, considerado de prognóstico mau devido a desidratação com evolução ao choque hipovolêmico do espécime de preguiça-comum atendido no HVSAS-UFPA. Mesmo com o uso de procedimentos ainda não padronizados para a espécie e ausentes na literatura foi possível a reabilitação e soltura do animal na natureza tornando-o aplicável em casos semelhantes nesta espécie.

\section{MANUFACTURERS}

${ }^{1}$ Farmace - Indústria Químico Farmacêutica Cearense Ltda. Barbalha, CE, Brazil.

${ }^{2}$ Vetoquinol USA Inc. Mairiporã, SP, Brazil.

${ }^{3}$ Halexistar - Indústria Farmacêutica S/A. Goiânia, GO, Brazil.

${ }^{4}$ Hipolabor Farmacêutica Ltda. Sabará, MG, Brazil.

${ }^{5}$ Hypofarma - Instituto de Hypodermia e Farmácia Ltda. Ribeirão das Neves, MG, Brazil.

${ }^{6}$ Isofarma - Industrial Farmacêutica Ltda. Eusébio, CE, Brazil.

${ }^{7}$ Novafarma - Indústria Farmacêutica. Anápolis, GO, Brazil.

${ }^{8}$ Lema Biologic do Brasil Ltda. São José dos Pinhais, PR, Brazil.

Declaration of interest. The authors report no conflicts of interest. The authors are responsible for content and writing of the paper.

\section{REFERENCES}

1 Ballesteros J., Reyes K. \& Racero J. 2009. Estructura poblacional y etología de Bradypus variegatus en fragmento de bosque seco tropical. Revista MVZ Córdoba. 14(3): 1812-1819.

2 Blasco P.G., Levites M.R., Mônaco C., Garcia D.S.O. \& Pinheiro T.R. 2009. Associação dexametasona oral e epinefrina inalatória é mais efetiva do que as intervenções isoladas de epinefrina ou dexametasona para o tratamento de bronquiolite. Diagnóstico e Tratamento. 14(4): 156-157.

3 Carvalho M.M., Pieri N.C.G., Pereira K.F., Lima F.C., Carniatto C.H.O., Miglino M.A., Ricci R.E. \& Martins D.S. 2014. Caracterização comparativa do intestino das espécies da Ordem Xenarthra. Pesquisa Veterinária Brasileira. 34: 49-56.

4 Castro-Vásquez L., Meza M., Plese T. \& Moreno-Mora S. 2010. Activity patterns, preference and use of floristic resources by Bradypus variegatus in a tropical dry forest fragment. Edentata. 11(1): 62-69.

5 Faro T.A.S., Lima A.R., Messias A.C., Cabral R., Giese E.G., Matos E.R. \& Branco E. 2015. Morfologia e morfometria da traqueia da preguiça (Bradypus variegatus): Conhecimentos para procedimentos de emergência. Pesquisa Veterinária Brasileira. 35: 193-198.

6 Ferreira R., Rezende C., Mortoza R., Martins S. \& Maria S. 2013. Modelo de suprimento sanguíneo do intestino delgado e grosso da preguiça de coleira. Ciência Rural. 43: 1114-1121.

7 Gilmore D.P., Da-Costa C.P. \& Duarte D.P.F. 2000. An update on the physiology of two- and three-toed sloths. Physiology of sloths. Brazilian Journal of Medical and Biological Research. 33: 129-146.

8 Gine G.A.F., Cassano C.R., Almeida S.S. \& Faria D. 2015. Activity budget, pattern and rhythm of maned sloths (Bradypus torquatus): responses to variations in ambient temperature. Mammalian Mammalian Biology-Zeitschrift für Säugetierkunde. 80(6): 459-467.

9 Hayssen V. 2010. Bradypus variegatus (Pilosa: Bradypodidae). Mammalian Species. 42(850): 19-32.

10 Javier V.D. \& Marrugo-Cano J.A. 2015. Primeros auxilios en decomisos de animales de venta o tráfico ilegal. Revista de Faculdade em Medicina. 63: 301-302.

11 Lichtenberger M. 2004. Principles of shock and fluid therapy in special species. Seminars in Avian and Exotic Pet Medicine. 13: 142-153.

12 Lichtenberger M. 2007. Shock and Cardiopulmonary-Cerebral Resuscitation in Small Mammals and Birds. Veterinary Clinics Exotic Animals. 10: 275-291.

13 Lima D.A.S.D., Lima W.C., Rodrigues M.C., Quessada A.M., Dos Santos K.M.M., De Moura C.R.C., Magalhães C.S. \& Sousa J.M. 2012. Trauma elétrico em preguiça de vida livre: relato de caso. Revista Portuguesa de Ciências Veterinárias. 111: 199-202.

14 Mannarino R.V. 2012. Obstrução respiratória alta em pediatria. Revista de Pediatria SOPERJ. 13: 54-60 
15 Martins D.M., Pinheiro L.L., Ferreira V.C., Costa A.M., Lima A.R., Ricci R.E.G., Miglino M.A. \& Branco E. 2014. Tongue papillae morphology of brown-throated sloth. Arquivo Brasileiro de Medicina Veterinária e Zootecnia. 66(5): 1479-1486.

16 Messias-Costa A. 2001. Medicine and neonatal care of sloths. In: Fowler M.E. \& Cubas Z.S. (Eds). Biology, Medicine and Surgery of South American Wild Animals. Ames: Iowa State University Press, pp.247-249.

17 Moreno S. \& Plese T. 2006. The illegal traffic in sloths and threats to their survival in Colombia. Edentata. 7: 10-18.

18 Mourão-Junior C.A. \& Souza L.S. 2014. Fisiopatologia do Choque. HU Revista. 40: 75-80.

19 Oliger C.D. \& Nicolai G.P. 2017. Manual de Manejo, Medicina y Rehabilitacion de Perezosos. Valdivia: Fundación Huálamo - Centro de Rehabilitación de Fauna Silvestre, 162p.

20 Pacheco M.A., Concepción J.L., Rangel J.D.R., Ruiz M.C., Michelangeli F. \& Domínguez-Bello M.G. 2007. Stomach lysozymes of the three-toed sloth (Bradypus variegatus), an arboreal folivore from the Neotropics. Comparative Biochemistry and Physiology. Part A. 147: 808-819.

21 Pedrosa M.A.C., Lima A.M.J., Bezerra A.P., Duarte D.P.F. \& Da-Costa C.P. 2002. The effect of feeding on the respiratory activity of the sloth. Brazilian Journal of Medical and Biological Research. (35): 851-854.

22 Puerto E. 2012. Causa de morte de animais silvestres oriundos de uma área de resgate: implicações na conservação. 197f. São Paulo, SP. Tese (Doutorado em Ciências) - Programa de Pós-Graduação em Patologia Experimental e Comparada, Universidade de São Paulo.

23 Riaño D.M.D, Villamizar N.P. \& Rico C.I.B. 2016. Casuística de perezosos (Bradypus variegatus y Choloepus hoffmanni) recibidos en la Unidad de Rescate y Rehabilitación de Animales Silvestres (URRAS) de la Universidad Nacional de Colombia entre 2005 y 2010. Edentata. 17: 34-40.

24 Rodrigues E., Avelar D., Alfredo L. \& Lopes M. 2011. Ameaças à Sobrevivência de Animais Silvestres no Estado de Goiás. Uniciências. 19(2): 132-140.

25 Silva E. \& Rocha A.M.C. 2015. Realização de cesariana em uma preguiça comum (Bradypus variegatus) de vida livre como tratamento emergencial de parto distócico. Sociedad Latinoamericana de Medicina de Emergencias e Cuidados Intensivos. 7(2): 30-42.

26 Silva G.A.O., Paz M.C.P. \& Cordeiro T.A. 2017. Monitoramento do bicho-preguiça Bradypus variegatus Schinz, 1825 (Xenarthra: Bradypodidae) em um remanescente de Floresta Atlântica (João Pessoa-PB, Nordeste do Brasil). Revista Brasileira de Gestão Ambiental e Sustentabilidade. 4: 299-312.

27 Toledo N., Bargo M.S. \& Vizcaíno S.F. 2012. Muscular Reconstruction and Functional Morphology of the Forelimb of Early Miocene Sloths (Xenarthra, Folivora) of Patagonia. Anatomy Research. 35: 296-305. 